\title{
Efficiency tests on a series of common skin antiseptics under ward conditions
}

\author{
P. E. VERDON \\ From the Portsmouth and Isle of Wight Pathological Service
}

SYNOPSIS Bacteriological trials were carried out on 11 different skin disinfecting agents to ascer tain their relative efficiencies under ward conditions. A statistical analysis of the results is given. Of the antiseptics used, $5 \%$ Laurolinium appeared to be the most effective and has been adopted for routine use in the wards.

In 1958, a survey of sterilizing methods was made in two of the hospitals of the Portsmouth Group before the introduction of a central sterile supply.

It was found that $60 \%$ of the sterile procedures carried out in the wards were the giving of hypodermic injections and that the techniques and skin disinfectants used varied considerably from one ward to another. This paper describes the investigations carried out to determine the efficiency of skin disinfection before injection under ward conditions.

\section{HISTORICAL}

In the past many of the tests made to evaluate skin disinfection were of a laboratory nature. Fleming in 1924, using the Wright 'slidecell' method, illustrated the germicidal and leucocyte-inactivating properties of blood and used it for evaluating a number of antiseptics. Another approach was to study the effect of antiseptics on the skin of animals in vivo and on egg membranes (Browning, 1934; Hunt, 1937; Green and Birkeland, 1944). These tests, however, were of little practical value in assessing the effects of disinfectants on human skin.

In his book 'Disinfection and Sterilization,' Sykes (1958) described a direct swabbing technique which he had found satisfactory. This tested the retention of activity by disinfectants other than soaps and had been adapted from that described by Colebrook and Maxted (1933). A similar method was proposed by Story (1952) in which small circles of skin on the forearm were infected with a drop from a chosen culture. Various handwashing tests have been published by different workers devised primarily for testing antiseptic soaps and, of these, that described by Cade (1950) seems to be most generally used. None of these investigations gave any indication as to the number of bacterial colonies likely to be found on the skin of patients, nor of the ability of disinfectants under ward conditions to reduce their numbers. In addition they did not simulate the actual procedure carried out by a nurse when swabbing the arm of a patient before giving an injection. In view of the fact that the nurse swabs the area of skin once or twice at the most, it was felt important to determine whether any of the antiseptics used in this way were effective.

Eleven antiseptics were chosen for trial, since they appeared to be those most commonly used in the hospitals where the tests were carried out. These were: Roccal 1 in 40, methylated ether, cetrimide $0.5 \%$ with Hibitane, $0.05 \%$ Penotrane, 1 in 20 dequalinium chloride, $0.5 \%$ merthiolate in aqueous solution, 1 in 1,000 tinct. merthiolate, 1 in 1,000 with $50 \%$ alcohol, solution of iodine $1 \%$ in $90 \%$ spirit, and Laurolinium $1 \%$ and $5 \%$.

\section{METHOD}

To determine the number of organisms on a given area of skin the technique of 'replica plating' was used (Lederberg and Lederberg, 1952). Replica plating was also used to provide a simple form of viable count by Elek and Hilson (1954). The advantages of this method are that a relatively large area of skin, 1 in. $\times 1$ in., can be tested and the transfer carries over very little more antiseptic than the amount adhering to individual organisms. The replica blocks used consisted of 1 in. $\times$ 1 in. $\times 1$ in. wooden blocks on one of whose surfaces was glued a square of velvet 1 in. $\times 1$ in. This provided the 'contact' area. One of these blocks could be used many times and was easily sterilized by steam. The portion of skin used for the tests was the same for every patient, namely, an area on the inner aspect of the arm 2 in. below the cubital fossa. The culture media used were nutrient agar for methylated ether, $1 \%$ iodine in $90 \%$ spirit, and $70 \%$ alcohol, and blood agar for all the other disinfectants. 
To carry out the trial, the block was first placed on the area B (shown below) of a culture medium plate to pick up a thin film of agar on its velvet surface. The block was then applied to the patient's arm to pick up bacteria and then pressed lightly back onto the same area of the plate. The arm was then swabbed quickly twice with a ball of sterile cotton wool dipped in the antiseptic solution being tested. Another identical sterile 'replica' block was taken and placed on square $A$ of the agar plate. It was then pressed lightly first onto the patient's arm over the swabbed area and then onto the same square.

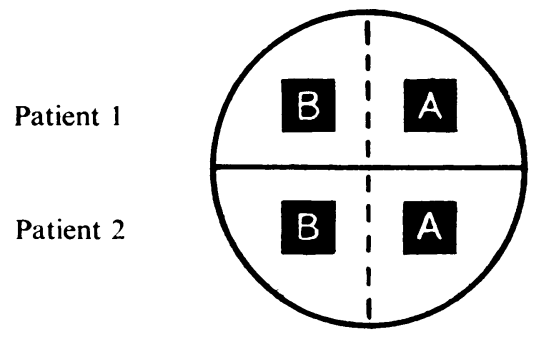

Diagram of an agar plate used in the test. One plate was used for each two patients.

This procedure was repeated on 100 different patients, male or female, for each solution under trial.

After 24 hours' incubation at $37^{\circ} \mathrm{C}$. the plates were examined and the number of colonies of bacteria on each square counted.

Certain possibilities have to be considered when carrying out these tests. Since the area of skin used for each of the two samples was the same, difficulty might have been experienced when interpreting the results because the bacterial population might, in fact, have been reduced by the first sampling, and the apparent reduction in colony count be due to this factor and not to the action of the particular antiseptic solution used in the test. In order to exclude this possibility a further 100 tests were carried out in which the same skin area was sampled twice without any intervening swabbing with antiseptic solution.

A further possibility also had to be taken into account when interpreting the results and that was 'carry-over' of the antiseptic fluid to the culture plate. Should this happen the counts would not reflect truly the immediate disinfecting powers of the solutions. This applied particularly to the slowerdrying aqueous solutions.

Supplementary investigations were, therefore, carried out on those solutions which were slower to volatilize by using culture medium to which a suitable inhibitor had been added, thus preventing continued action by any of the antiseptic carried over. These supplementary tests were carried out on both of the Laurolinium solutions and the cetrimide-Hibitane mixture, since from early results it appeared that these solutions had not completely $\underline{0}$ evaporated before the skin was resampled after swabbing. $\Rightarrow$ The inhibitor used was $1 \%$ lubrol W with $0.5 \%$ lecithin.

For each of these tests four blocks and two blood agar 0 plates, to one of which the inhibitor had been added, were $\frac{\bar{\sigma}}{\square}$ used. Samples were taken simultaneously from two $\overline{\frac{\bar{\sigma}}{5}}$. adjacent sites on the routine test area of the arm and $\vec{\nabla}$ plated out onto square B of the two different culture $\varrho$ plates. Both skin areas were then swabbed with the test $\stackrel{\text { \% }}{\circ}$ solution and a further two blocks applied to them and $\vec{\circ}$ again pressed onto the two areas $\mathrm{A}$ of each plate.

\section{RESULTS}

It was found that taking two consecutive samples from the same area of untreated skin without intervening swabbing slightly reduced the number of 0 colonies isolated from the second replica block when $\vec{\circ}$ compared with the first, but the reduction was not statistically significant. The counts on inhibitor and $\overrightarrow{\vec{c}}$ non-inhibitor culture plates also showed no significant difference. These two possibilities can, therefore, be discounted when assessing the relative efficiency of the different disinfecting fluids.

The table summarizes the results of the plate counts before and after the application of the various fluids to the skin and gives their statistical sig-O nificance.

Because the number of colonies counted showed such a wide range from 0 to more than 100 , with the $\frac{0}{\varnothing}$ smaller counts predominating, the actual figures ob- $\varrho$ tained were replaced by their square roots, as shown $\overrightarrow{\overrightarrow{0}}$ in column 3 of the table. These figures were used in 3 the analysis. For example, if the number of colonies counted was 100 or more, the square root was taken? as 10. This gave a more even distribution and madêㅇ the statistical analysis more likely to be correct. The figures in column 3 show the mean decrease in terms of the square root of the number of colonies afteri swabbing. The antiseptic solutions are placed in the order of their apparent effectiveness.

\section{DISCUSSION}

The disinfecting agents fall into five groups, showingo different degrees of effectiveness. Within the individual groups the solutions appear fairly uniform in their action.

The tests of significance given in columns 4 and $5^{0}$ of the Table show that with the exception of Peno는 trane and aqueous merthiolate (group I) all the solu䨌 tions had a significantly destructive effect on the skin flora. Methylated ether (group II), though better than the group I reagents, was relatively ineffective $\vec{D}$ Dequalinium chloride and $70 \%$ alcohol (group III were significantly more potent than the group IP solutions. 


\section{TABLE}

\section{RESULTS OF COMPARISON OF 11 SKIN DISINFECTING AGENTS}

(1) Skin Sterilizing Agents

(2) No. of Patients

(3) Mean Decrease in Square Root of crease Compared with that for No No. of Colonies Agent after Swabbing
(5) Statistical Significance o Decrease Compared with that for Last Preceding Agents
GROUP I

None (control test)

Penotrane stock solution 1:20

Merthiolate (aqueous) 1 in 1,000

GROUP II

Methylated ether

GROUP III

Dequalinium chloride 0.5\%

Alcohol 70\%

GROUP IV

Iodine $1 \%$ in spirit $90 \%$

Merthiolate (tincture) 1 in 1,000

Cetrimide $0.5 \%$ and Hibitane $0.05 \%$

Roccal (benzalkonium) 1 in 40

GROUP V

Laurolinium $1 \%$

Laurolinium $5 \%$

$\begin{array}{ll}100 & 0 \cdot 18 \\ 100 & 0 \cdot 29 \\ 100 & 0 \cdot 34 \\ 100 & \\ & 0 \cdot 82 \\ 100 & \\ 100 & 1 \cdot 47 \\ & 1 \cdot 56 \\ 100 & \\ 100 & 2 \cdot 02 \\ 100 & 2 \cdot 18 \\ 100 & 2 \cdot 1 \\ & 2 \cdot 22 \\ 100 & \\ 100 & 3 \cdot 16 \\ & 3 \cdot 38\end{array}$

$\mathbf{P}=$ statistical significance of decrease.

The agents in group IV were not significantly more active than the $70 \%$ alcohol of group III, but the two Laurolinium solutions in group $\mathrm{V}$ were much the most efficient of all the skin disinfecting agents tested.

It is appreciated that we have no definite proof that lubrols $\mathbf{W}$ and lecithin will inhibit Laurolinium in the concentrations used for the trials.

In view of these findings, $5 \%$ Laurolinium has now been adopted as the standard pre-injection skin disinfectant in all wards in the Portsmouth Group which are served by the Central Sterile Supply Department.

My thanks are due to Dr. E. M. Darmady and Dr. K. E. A. Hughes, of the Central Laboratory, Portsmouth, for their advice and encouragement. I wish particularly to thank Dr. Ian Sutherland, of the Medical Research Council Laboratories, Hampstead, for his advice and for carrying out all the statistical work on the bacterial counts. Tharks are also extended to Dr. L. L. Banks, of Allen \& Hanburys Clinical Trials Department, who supplied the samples of Laurolinium, and lastly to Mr. J. Jones and Mr. D. Prince for their technical assistance.

\section{REFERENCES}

Browning, C. H. (1934). Brit. med. J., $2,579$.

Cade, A. R. (1950). Soap, 26 (7), p. 35.

Colebrook, L., and Maxted, W. R. (1933). J. Obstet. Gynaec. Brit Emp., 40, 966.

Elek, S. V., and Hilson, G. R. F. (1954), J. clin. Path., 7, 37.

Fleming, A. (1924). Proc. roy. Soc. B., 96, 171.

Green, T. W., and Birkeland, J. M. (1944). J. infect. Dis., 74, 32.

Hunt, G. A. (1937). Ibid., 60, 232.

Lederberg, J., and Lederberg, E. M. (1952). J. Bact., 63, 399.

Story, P. (1952). Brit. med. J., 2, 1128.

Sykes, G. (1958). Disinfection and Sterilization. Spon, London. 